\title{
A role for neural determination genes in specifying the dorsoventral identity of telencephalic neurons
}

\author{
Carol Fode, ${ }^{1}$ Qiufu Ma, ${ }^{2,3}$ Simona Casarosa, ${ }^{1}$ Siew-Lan Ang, ${ }^{1}$ David J. Anderson, ${ }^{2}$ \\ and François Guillemot ${ }^{1,4}$ \\ ${ }^{1}$ Institut de Génétique et de Biologie Moléculaire et Cellulaire, Centre National de la Recherche Scientífique/Institut \\ National de la Santé et de la Recherche Médicale (IGBMC, CNRS/INSERM); Université Louis Pasteur, BP163, Communauté \\ Urbaine de Strasbourg, France; ${ }^{2}$ Howard Hughes Medical Institute, Division of Biology 216-76, California Institute \\ of Technology, Pasadena, California 91125 USA
}

\begin{abstract}
Neurogenin1 (Ngn1), Neurogenin2 (Ngn2), and Mash1 encode bHLH transcription factors with neuronal determination functions. In the telencephalon, the Ngns and Mash1 are expressed at high levels in complementary dorsal and ventral domains, respectively. We found that Ngn function is required to maintain these two separate expression domains, as Mash1 expression is up-regulated in the dorsal telencephalon of Ngn mutant embryos. We have taken advantage of the replacement of the Ngns by Mash1 in dorsal progenitors to address the role of the neural determination genes in neuronal-type specification in the telencephalon. In Ngn2 single and Ngn1; Ngn2 double mutants, a population of early born cortical neurons lose expression of dorsal-specific markers and ectopically express a subset of ventral telencephalic-specific markers. Analysis of Mash1; Ngn2 double mutant embryos and of embryos carrying a Ngn2 to Mash1 replacement mutation demonstrated that ectopic expression of Mash1 is required and sufficient to confer these ventral characteristics to cortical neurons. Our results indicate that in addition to acting as neuronal determinants, Mash1 and Ngns play a role in the specification of dorsal-ventral neuronal identity, directly linking pathways of neurogenesis and regional patterning in the forebrain.
\end{abstract}

[Key Words: Cerebral cortex; thalamus; neurogenesis; mouse mutants; Mash1; neurogenin; bHLH gene]

Received August 9, 1999; revised version accepted November 19, 1999.

The molecular events controlling the generation of specific neuronal types at precise positions and in appropriate numbers in the neural tube are beginning to be deciphered. A major advance in our understanding of this process came from morphological and molecular marker analyses, which revealed that the neural tube is subdivided into transverse and longitudinal domains from early embryonic stages. In particular, it has been proposed that the forebrain neuroepithelium can be subdivided into six prosomeric units (Puelles and Rubenstein 1993). An important characteristic of the forebrain prosomeres is that progenitor cell mixing is prevented across their boundaries (Figdor and Stern 1993; Neyt et al. 1997), a restriction that is required for the subsequent generation of anatomically and functionally distinct compartments in the adult brain.

The six prosomeric units of the forebrain are transverse domains that can be further subdivided longitudi-

Present address: ${ }^{3}$ Dana-Farber Cancer Institute, Department of Neurobiology, Harvard Medical School, Boston, Massachusetts 02115 USA ${ }^{4}$ Corresponding author.

E-MAIL francois@igbmc.u-strasbg.fr; FAX (33)3-88-65-32-01. nally by the restricted expression patterns of many regulatory molecules (for review, see Puelles and Rubenstein 1993). In the embryonic telencephalon, which gives rise to the cerebral cortex dorsally and the basal ganglia ventrally, homeobox genes of the empty spiracles (Emx1, Emx2) and paired homeobox (Pax6) families are expressed exclusively in dorsal progenitors, whereas ventral progenitors express homeobox genes of the $N k x$ (Nkx2.1) and distal-less $(D 1 \times 1, D l \times 2)$ families (for review, see Rubenstein et al. 1998). Postmitotic neurons also have distinct molecular phenotypes in these two subdivisions of the telencephalon. For example, GAD67, which encodes the enzyme required for the biosynthesis of the GABA neurotransmitter, is expressed by ventral telencephalic neurons of the basal ganglia, whereas the bHLH transcription factors NeuroD, Math2, Nscl1, and Nscl2 and the T-box gene Tbr1 (Bulfone et al. 1995; Lee 1997) are expressed only in dorsally derived neurons of the cerebral cortex. Although the genetic networks leading to the generation of distinct neuronal phenotypes, cytoarchitecture, and axonal connections remain for the most part unknown, the acquisition of different molecular identities by dorsal and ventral telencephalic progeni- 
tors is likely the basis for the later diversification of neuronal types between the cerebral cortex and basal ganglia (for review, see Fishell 1997).

An important consideration regarding the specification of neuronal identities is that the pathways underlying cell-type specification must be coordinated with genetic programs that impart generic neuronal properties (for review, see Anderson and Jan 1997; Edlund and Jessell 1999). In Drosophila, the proneural genes, which include genes of the achaete-scute complex $(a s-c)$ and atonal (ato), confer both panneuronal properties and neuronal subtype-specific identities to neural precursors (for review, see Jan and Jan 1994). Gain-of-function analyses indicate that in addition to acting as neuronal determinants, $a s-c$ genes play a role in the specification of external sensory organs, whereas ato promotes chordotonal organ development (Chien et al. 1996; Jarman and Ahmed 1998). In addition, loss-of-function analyses and rescue experiments indicate that $a s-c$ genes regulate some aspects of neuronal phenotype identity in CNS precursors (Parras et al. 1996; Skeath and Doe 1996).

In vertebrates, the ato-related genes Ngn1 and Ngn2 (Ngns) and the as-c-related gene Mash1 have neural determination functions in cranial sensory lineages (Fode et al. 1998; Ma et al. 1998), and in peripheral autonomic lineages, olfactory epithelium, and ventral forebrain (Cau et al. 1997; Lo et al. 1997; Casarosa et al. 1999; Torii et al. 1999), respectively. In addition, the spatially restricted and largely nonoverlapping patterns of expression of Mash1 and the Ngns (Gradwohl et al. 1996; Ma et al. 1997) suggest that they may also play a role in the specification of distinct neuronal identities. In support of this hypothesis, it has been demonstrated that Mash1 is required for the differentiation of a noradrenergic phenotype in hindbrain and PNS neurons, acting through the activation of, or in concert with, the Phox2 homeodomain transcription factors (Hirsch et al. 1998; Lo et al. 1998; for review, see Goridis and Brunet 1999|. Loss-offunction and gain-of-function experiments have also indicated that Mash1 and the ato-related gene NeuroD control the ratio of interneurons of distinct classes in the retina (Tomita et al. 1996; for review, see Cepko 1999; Morrow et al. 1999). Finally, it has been demonstrated that in the chick, the Ngns can induce the expression of panneuronal as well as sensory-specific markers in neural crest cells, and in the dermomyotome, a mesodermal tissue (Perez et al. 1999).

In the telencephalon, Mash1 is expressed at high levels by ventral progenitors, whereas Ngn1 and Ngn2 are expressed exclusively in cells of the dorsal ventricular zone (VZ) (Gradwohl et al. 1996; Sommer et al. 1996; Ma et al. 1997). Here, we have addressed the question of whether the restricted patterns of expression of Mash1 and the $N g n s$ in the telencephalon reflect a role for these genes in the specification of distinct neuronal identities in the basal ganglia and cerebral cortex, respectively. We show that Ngn gene function is required to prevent ectopic Mash1 expression in the dorsal telencephalon, ensuring that the pallial and subpallial subdivisions of the telencephalon express different neural determination genes.
The up-regulation of Mash1 in the dorsal telencephalon compensates to some extent for the loss of the determination function of the Ngns, as neurogenesis defects in Ngn mutant cortices are mild, as compared with the more severe defects in the Ngn2;Mash1 mutant cortex. However, the importance of restricting Mash1 expression to the ventral telencephalon for correct neuronal patterning is highlighted by the observation that ectopic Mash1 expression in Ngn mutants results in the misspecification of a subpopulation of early born cortical neurons, which lose expression of several dorsal markers (e.g., Math2, Nsc11, Nsc12, Tbr1) and ectopically express the ventral markers Dlx1, Dlx2, Dlx5, and GAD67. These data are supported by the results of forced expression of Mash1 in dorsal telencephalic progenitors, which is sufficient to confer ventral characteristics to a subset of cortical neurons. These results indicate that Mash1 and the Ngns are involved in coupling neural determination and the specification of neuronal phenotypes during forebrain development.

\section{Results}

Complementary expression of Ngns and Mash1 in the telencephalon

Previous expression studies have shown that Mash1 and the Ngns are expressed in a complementary manner in the developing telencephalon and diencephalon, suggesting that these genes may play a role in specifying regional neuronal identity in the forebrain (Gradwohl et al. 1996; Ma et al. 1997). To begin to investigate this hypothesis, we compared the expression patterns of these genes in greater detail. To better trace Ngn2-expressing progenitors, a new null allele of $N g n 2\left(N g n 2^{\text {lacZKI}}\right)$, in which coding sequences of the bHLH domain were replaced with a lac $Z$ reporter, was introduced in mice by standard embryonic stem (ES) cell technology (Fig. 1A). Loss of most Ngn2 coding sequences, including the bHLH domain, in Ngn2 $2^{l a c Z K I}$ homozygous embryos was confirmed by Southern blot (Fig. 1A) and PCR analysis (data not shown). The Ngn2 ${ }^{\text {lacZKI }}$ allele behaves as a null allele on the basis of the identical perinatal lethality and cortical phenotype observed in Ngn2 $2^{l a c Z K I}$ and $N g n 2^{\text {neo }}$ null homozygous embryos (Fode et al. 1998; this paper; data not shown).

At embryonic day (E) 12.5, transcripts for Ngn1 (Fig. 1B) and Ngn2 (Fig. 1C) are restricted to the VZ of the dorsal telencephalon, showing a sharp extinction of expression at the border with the ventral telencephalon. $N g n 1$ is expressed in a graded manner, with higher levels in the lateral VZ, whereas Ngn2 transcripts appear distributed more uniformly throughout the dorsal VZ. Staining of Ngn2 $2^{\text {lacZKI }}$ heterozygous embryos at E12.5 with $\mathrm{X}$-Gal indicated that $\beta$-galactosidase activity is detected in similar restricted domains of the forebrain as $N g n 2$ transcripts, including the dorsal telencephalon and dorsal thalamus (Fig. 1D). However, $\beta$-galactosidase activity is detected in a gradient in the VZ of the telencephalon, with higher levels in dorsomedial progenitors 
A
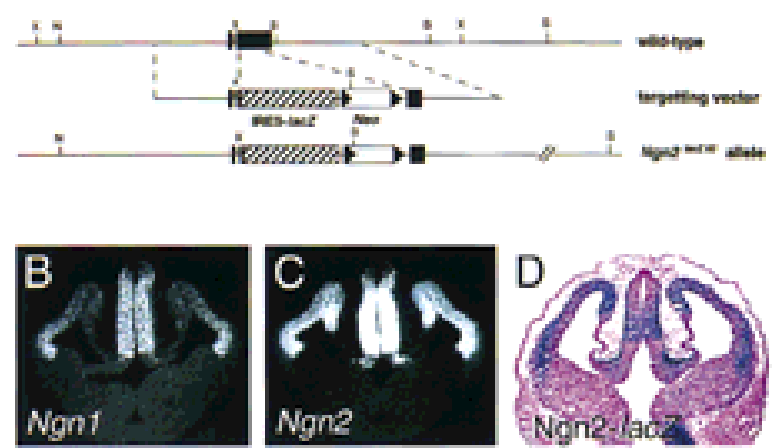

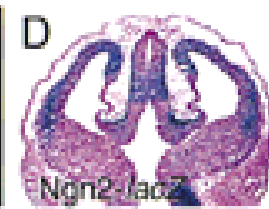

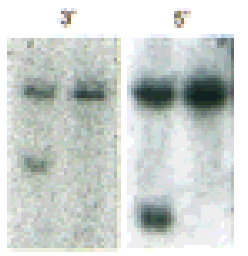

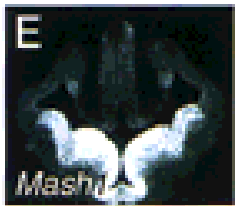

Figure 1. Mash1 and Ngns are expressed in complementary patterns in the embryonic telencephalon. (A) A new Ngn2 null allele was generated by replacing the sequence encoding the bHLH domain with the lacZ reporter $\left(N g n 2^{1 a c Z K I}\right)$. Schematic representation of the endogenous Ngn2 locus (top), Ngn2 targeting vector (middle), and recombined allele (bottom). Southern blot analysis of NotI-SpeI-digested ES cell genomic DNA with 5' and 3' external probes that recognize a common 20-kb wild-type band and 7.5- and 10.5 -kb mutant bands, respectively. $(B, C, E)$ Distribution of transcripts for Ngn1 $(B), N g n 2(C)$ and Mash1 (E) on frontal sections through the forebrain of E12.5 embryos. (D) X-gal staining of Ngn2lacZKI heterozygous embryos at E12.5. Ngn1 and Ngn2 are expressed in proliferating progenitors of the dorsal telencephalon and dorsal thalamus in an almost completely overlapping fashion. Mash1 is expressed in a complementary manner with transcripts at high levels in the VZ of the ventral telencephalon and ventral thalamus.

(Fig. 1D) in comparison with the more uniform distribution of Ngn2 transcripts. In addition, $\beta$-galactosidase activity is maintained in postmitotic neurons accumulating throughout the preplate, serving as a short-term lineage tracer and allowing us to conclude that Ngn2derived neurons are present throughout the lateral to medial extent of the cortex.

In contrast to Ngns, Mash1 is expressed at high levels in ventral progenitors located in the lateral (LGE) and medial ganglionic eminences (MGE) and only at low levels in the dorsal telencephalon (Fig. 1E). The complementary patterns of expression of these genes are also observed in the dorsal thalamus, in which Ngn1 (Fig. 1B) and Ngn2 (Fig. 1C) but not Mash1 (Fig. 1E), are expressed at high levels.

\section{Ngns down-regulate Mash1 expression in dorsal} telencephalon and dorsal thalamus

Cross-regulatory interactions exist between $N g n 1$ and $N g n 2$ in neuronal lineages of the PNS, including the dor- sal root and cranial ganglia (Fode et al. 1998; Ma et al. 1998, 1999). Thus, we examined whether the overlap in expression of Ngn1 and Ngn2 in the telencephalon reflects cross activation or independent means of activation, by studying $N g n 1$ expression in $N g n 2$ mutants and vice versa. In the dorsal telencephalon of Ngn2 mutants, Ngn1 expression is lost in medial and dorsal domains but is maintained in the lateral VZ (Fig. 2B). In contrast, $N g n 2$ expression is detected throughout the dorsal telencephalon of Ngn1 mutants, although the levels appear reduced (Fig. 2D). Thus, regulatory interactions between the Ngns exist in the forebrain, such that Ngn2 mutant progenitors in the dorsal/medial cortex fail to express both Ngn1 and Ngn2, and Ngn1 mutant progenitors express reduced levels of Ngn2 throughout the dorsal telencephalon.

We also investigated potential regulatory interactions between Mash1 and the Ngns in the telencephalon. An up-regulation of Mash1 expression was observed throughout the dorsomedial cortical VZ of Ngn2 mutant embryos at E12.5 (Fig. 2G) and later stages (E13.5; Fig. 2J).
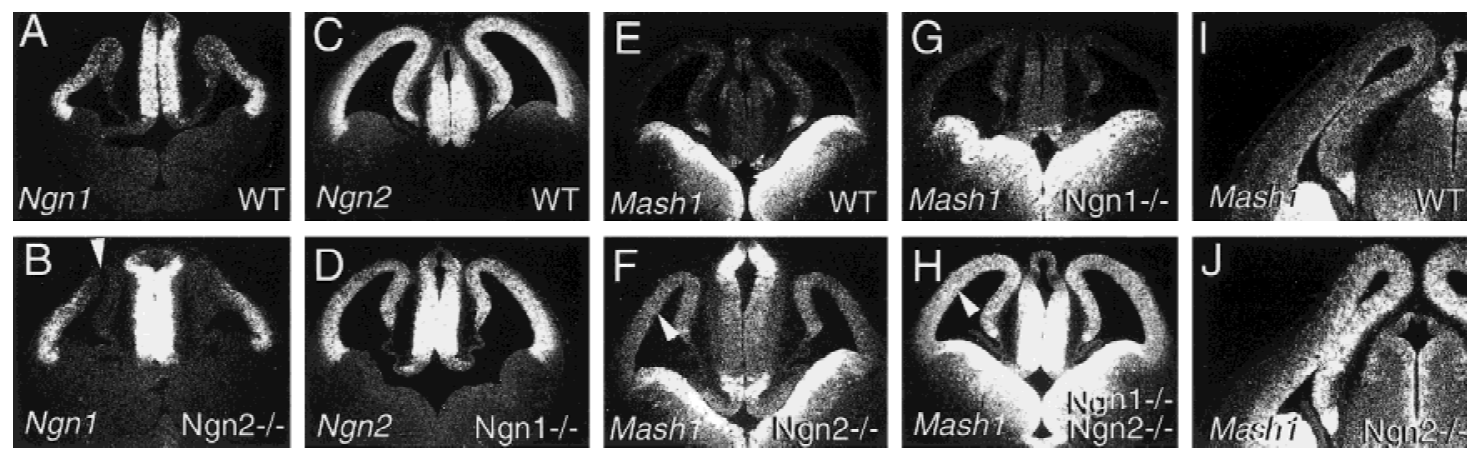
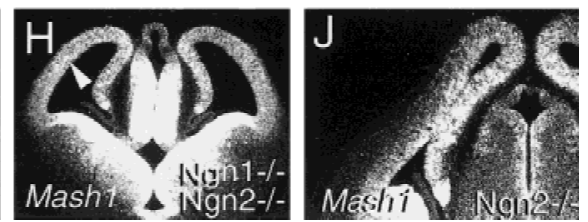

Figure 2. Ngns down-regulate Mash1 transcription in the dorsal telencephalon. $(A, B)$ Distribution of Ngn1 transcripts on frontal sections of E12.5 wild-type $(A)$ and Ngn2 mutant $(B)$ forebrain. Ngn1 expression is dependent on Ngn2 function in dorsal and medial cortical domains (arrowhead, $B$ ) and independent of $N g n 2$ in a lateral cortical domain. $(C, D)$ Distribution of $N g n 2$ transcripts on frontal sections of E12.5 wild-type $(C)$ and Ngn1 mutant $(D)$ forebrain. Ngn2 is expressed at reduced levels in cortical progenitors of Ngn1 mutants, suggesting that Ngn2 transcription is partially dependent on Ngn1 function throughout the dorsal telencephalon. (E, $)$ Distribution of Mash1 transcripts on frontal sections of E12.5 wild-type (E), Ngn1 mutant $(G)$, Ngn2 mutant (G), and Ngn1; Ngn2 double mutant (H), and of E13.5 wild-type $(I)$ and Ngn2 mutant $(J)$ forebrain. Mash1 expression is up-regulated in the VZ of the dorsal telencephalon (arrowhead in Fand H) and dorsal thalamus of Ngn2 single and more strikingly of Ngn1; Ngn2 double mutant embryos. 
This up-regulation was less apparent in the lateral VZ, in which Ngn1 expression is maintained in Ngn2 mutants. No changes in Mash1 expression levels were observed in the forebrain of Ngn1 single mutants (Fig. 2F; data not shown), but Mash1 up-regulation was strikingly enhanced in the dorsal telencephalon and dorsal thalamus of Ngn1;Ngn2 double mutant embryos (Fig. 2H,K). In contrast, Ngn1 and Ngn2 expression patterns were unaltered in the forebrain of Mash1 mutant embryos /data not shown). Taken together, these results suggest that the Ngns function in a partially redundant manner to restrict Mash1 expression to limited domains of the forebrain neuroepithelium. The nature of the regulatory in- teractions between Ngns and Mash1 remains to be characterized.

\section{Dorsal neurons down-regulate dorsal markers} in Ngn mutant telencephalon

Given that the Ngns function as determination genes for PNS neurons (Fode et al. 1998; Ma et al. 1998), their mutation may result in neurogenesis defects in the dorsal telencephalon. To address this possibility, expression of the panneuronal marker SCG10 was examined at E12.5 in the telencephalic preplate (PP), the layer of earliest born cortical neurons. SCG10 expression is not sig-
Figure 3. Cortical preplate neurons lose dorsal characteristics and acquire ventral properties in Ngn mutants. $(A-D)$ Distribution of SCG10 transcripts on frontal sections of E12.5 wild-type and Ngn mutant telencephalon. SCG $10^{+}$neurons are found in the telencephalic PP of wild-type embryos $(A), N g n 1$ mutants $(B), N g n 2 \mathrm{mu}-$ tants $(C)$, and Ngn1;Ngn2 double mutants $(D) .(E-L)$ Distribution of Tbr1 transcripts $(E-H)$ and Math2 transcripts $(I-L)$ on frontal sections of E12.5 wild-type and Ngn1 mutant telencephalon, revealing a strong reduction in the expression of these dorsal-specific markers by postmitotic neurons of the dorsal and medial cortex in Ngn2 mutants (arrows, $G$ and $K$, respectively) and in lateral cortex of Ngn1;Ngn2 double mutants (arrows, $H$ and $L$, respectively). $(M-P)$ Distribution of Dlx1 transcripts on frontal sections of E12.5 wild-type and Ngn mutant telencephalon. This ventral marker is ectopically expressed by PP neurons in the dorsal cortex of Ngn2 mutant embryos (arrow, O) and in the dorsal and lateral cortex of Ngn1;Ngn2 double mutant embryos (arrow, $P) .(Q, R)$ Expression of Dlx1 in the telencephalon of E13.5 wild-type (Q) and Ngn2 mutant $(R)$ embryos, demonstrating that the ectopic expression of $D l_{x} 1$ has expanded to the medial and lateral cortex in $N g n 2$ mutant at this stage (arrow, $R)$. $(S-V)$ Expression of Dlx5 $(S, T)$ and GAD67 $(U, V)$ in the telencephalon of E13.5 wild-type and Ngn2 mutant embryos, demonstrating that these genes are also ectopically expressed in Ngn2 mutant cortex at this stage (arrow, $T$ and $V$, respectively). High-magnification views of Math2 (purple) and Dlx1 (orange) expression in the dorsal cortex of E13.5 wild-type (W) and Ngn2 mutant (X) embryos. Math2 and Dlx1 are not coexpressed in the Ngn2 mutant cortex, indicating that mutant PP neurons adopt either a dorsal or a ventral fate. The same misspecification of cortical neurons was observed in

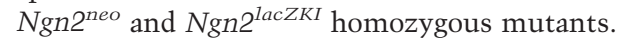

E12.5
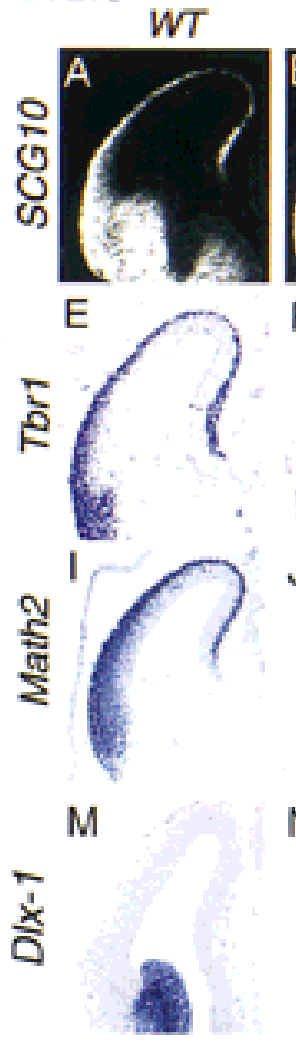

$\mathrm{N}$

E13.5
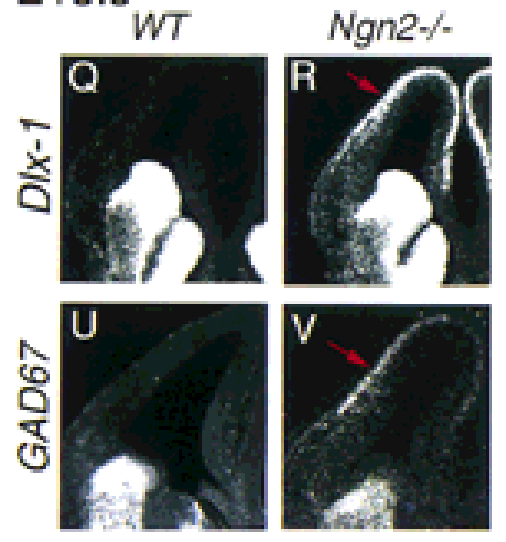

$\mathrm{F}$

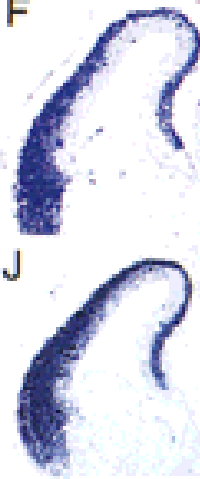

Ngn $1 \%$ $\mathrm{Ngn} 2 \%$
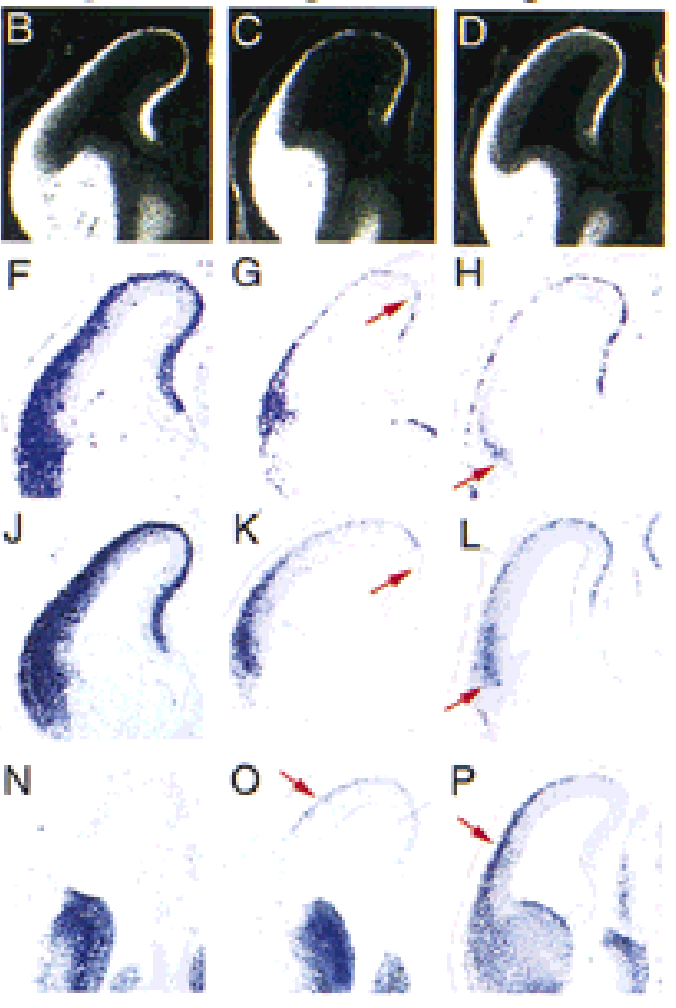

G

$\mathrm{H}$

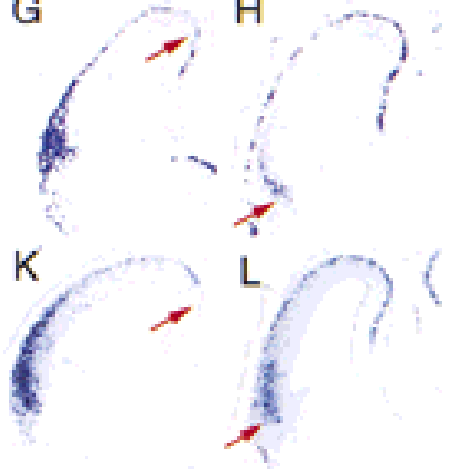

O
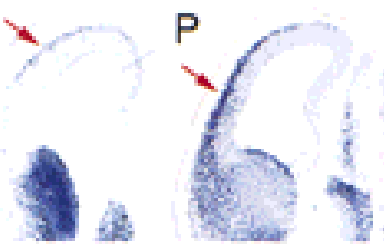

WT $\mathrm{s}$
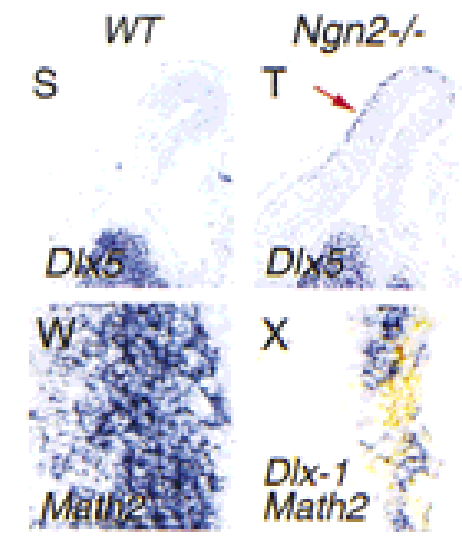
nificantly different in the PP of wild-type embryos and Ngn2 single mutants (Fig. 3A,C). In Ngn1 single (Fig. 3B) or Ngn1;Ngn2 double (Fig. 3D) mutant embryos, there is an apparent increase in the number of SCG10-expressing cells, a phenotype for which we have not yet identified a cause. However, the presence of neurons in the PP of Ngn mutants suggested that up-regulation of Mash1 could partially compensate for the lack of Ngn activity and permit cortical neurogenesis to proceed.

To examine whether the replacement of Ngns by Mash1 as a determination gene in Ngn mutant cortical progenitors resulted in specification defects, we first analyzed the expression of several dorsal-specific neuronal markers in the telencephalon of Ngn mutants. Reductions in expression levels of Tbr1 (Fig. 3G), Math2 (Fig. $3 \mathrm{~K}$ ), Nscl1, and Nscl2 (data not shown) were observed in the dorsal and medial PP of Ngn2 mutants at E12.5. Tbr1 and Math2 expression levels were not reduced in the PP of Ngn1 single mutants (Fig. 3F,J), but expression levels of these genes were further reduced in the lateral cortex of Ngn1;Ngn2 double mutants (Fig. 3H,L).

In contrast to the dorsal postmitotic markers, no changes were observed in the level of expression of dorsal-specific progenitor markers, including $E m \times 1, E m \times 2$, and Pax6, in the Ngn2 mutant cortex (data not shown), suggesting that with the exception of Mash1 up-regulation, the molecular phenotype of dorsal progenitors is unaltered in Ngn2 mutants.

\section{Dorsal neurons ectopically express ventral markers in Ngn mutant telencephalon}

We hypothesized that the down-regulation of dorsal-specific markers by Ngn mutant cortical neurons might reflect their respecification to a more ventral identity. To address this possibility, the expression patterns of several ventral telencephalic-specific genes were examined in $\mathrm{Ngn}$ mutant embryos. In wild-type E12.5 embryos, the closely related homeobox genes $D l \times 1$ and $D l \times 2$ are expressed at high levels in the VZ and SVZ of the ventral telencephalon, whereas their transcripts are not detected in dorsal domains (Figs. 3M and 6J, below, data not shown). Ectopic Dlx1 and Dlx2 expression was detected in the Ngn2 mutant cortex, beginning at E12 in the rostral and dorsal cortex (E12.5; Fig. 3O; data not shown), and progressing subsequently to more medial and caudal positions (E13.5; Fig. 3R) in a manner reflecting the neurogenic gradient. Ectopic Dlx1 expression was restricted to the neuronal layer and was not detected in the lateral cortex of Ngn2 single mutants, in which Ngn1 expression is maintained, nor in the cortex of $\mathrm{Ngn} 1$ single mutants, which maintain Ngn2 expression (Fig. 3N). However, an expansion of ectopic Dlx1 expression into the lateral and caudal cortex was observed in Ngn1;Ngn2 double mutants at E12.5 (Fig. 3P). By E13.5, additional ventral-specific genes, such as Dlx5 (Fig. 3T) and GAD67 (Fig. 3V), which encode a biosynthetic enzyme for the GABA neurotransmitter, were ectopically expressed by cortical neurons in Ngn2 single mutants. Several other ventral telencephalic markers, such as $L h \times 6, L h x 7$, Is- let1, and Nkx2.1, were not ectopically expressed in $N g n$ mutant cortical neurons, suggesting that these neurons are either only partially ventralized or have activated a subtype-specific ventral differentiation program (data not shown). Consistent with the idea that the decreased expression of dorsal markers correlates with a change to a ventral-like fate by Ngn mutant cortical neurons, double-labeling experiments revealed that Dlx1 and Math2 are expressed in largely nonoverlapping and complementary populations of neurons in the Ngn2 mutant PP (Fig. 3P).

\section{Changes in neuronal identity in the dorsal thalamus of Ngn mutants}

To determine whether neural $b H L H$ genes are involved in specification of neuronal phenotypes throughout the forebrain, we examined neuronal patterning in the thalamus, a region in which Mash1 and the Ngns are also expressed in a complementary manner. Ngn1 and Ngn2 are highly expressed in the dorsal thalamus (Fig. 1B,C) whereas Mash1 expression is restricted to the ventral thalamus (Fig. 4A). Ectopic Mash1 expression was observed in the dorsal thalamus of $N g n 2$ mutants (Fig. 4B) and more extensively in Ngn1;Ngn2 double mutant embryos, in which Mash1 transcripts were detected at high levels throughout the different subdivisions of the dien-

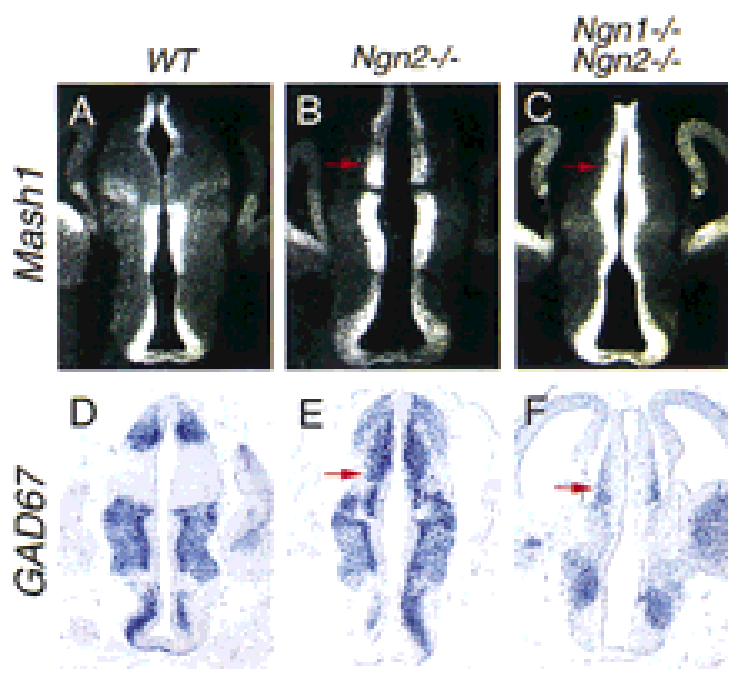

Figure 4. Mis-specification of neurons in the dorsal thalamus of Ngn mutants. $(A-C)$ Distribution of Mash1 transcripts on frontal sections of E12.5 wild-type and Ngn mutant diencephalon, showing that Mash1 expression is up-regulated in the dorsal thalamus of Ngn2 single mutants (arrow, B) and more extensively in Ngn1;Ngn2 double mutants (arrow, C). Distribution of GAD 67 transcripts on frontal sections of E12.5 wild-type and Ngn mutant diencephalon, demonstrating an alteration in neuronal identity in the dorsal thalamus of Ngn2 single and Ngn1;Ngn2 double mutants revealed by the ectopic expression of GAD67 (arrows, E, F). Morphological defects are also observed in the thalamus of Ngn1;Ngn2 double mutant embryos $(F)$, consistent with a role for the Ngn genes in neural determination in this region of the forebrain. 
cephalon (Fig. 4C). Therefore, the Ngns function to restrict Mash1 expression to limited domains throughout the forebrain neuroepithelium. In addition, consistent with the idea that altered neuronal identities arise in regions where Mash1 expression levels are up-regulated, ectopic GAD67 transcripts were detected in postmitotic neurons in the dorsal thalamus of Ngn2 single mutants (Fig. 4E). The mis-specification of neurons is more severe in Ngn1;Ngn2 double mutant embryos, because in addition to GAD67 (Fig. 4F), Dlx1 is also ectopically expressed in the dorsal thalamus (data not shown). Taken together with our observations in the cortex, these results indicate that the proper expression of $N g n$ and Mash1 is required for the correct specification of neuronal identity in several subdivisions of the forebrain. In addition, the dorsal thalamus of Ngn1;Ngn2 mutant embryos appears highly abnormal, with an enlargement of the VZ and a reduction in size of the mantle zone (Fig. $4 \mathrm{~F})$, suggestive of severe neurogenesis defects.

\section{Ectopic Dlx1 neurons are derived} from dorsal progenitors

The presence of cortical neurons with a ventral phenotype in Ngn mutants may arise from the mis-specification of dorsal progenitors or, alternatively, from premature and excessive tangential migration of ventrally derived neurons. In particular, a population of $D l \times 1^{+}$, $G A D 67^{+}$interneurons derived from ventral precursors has been shown to migrate tangentially from the ganglionic eminences into the dorsal telencephalon beginning at E13.5 (De Carlos et al. 1996; Anderson et al. 1997). We thus used several approaches to determine whether the ectopic Dlx1+ neurons observed in Ngn2 mutant cortex are dorsal or ventral in origin. As a first step to trace the origin of these neurons, we performed double-labeling experiments, using $\beta$-galactosidase activity in $N g n 2^{\text {lacZKI }}$ homozygous mutant embryos to mark neurons derived from Ngn2-expressing mutant progenitors, and thus of dorsal origin (Fig. 1D), followed by Dlx1 RNA in situ hybridization. Cortical neurons labeled with both X-Gal and Dlx1 were detected (Fig. 5A; arrows in B), indicating that at least some of these neurons are derived from Ngn2-expressing progenitors in the dorsal telencephalon.

To provide further support for the hypothesis that ectopic Dlx1-expressing cells arise from cortical progenitors, explant experiments were performed in which the dorsal telencephalon was separated from ventral territories at $\mathrm{E} 11$, prior to the appearance of $D 1 \times 1+$ neurons in the Ngn2 mutant cortex. Medial, dorsal, and ventrolateral domains were dissected out and cultured separately on filters for 4 days (Fig. 5A). After culture, clusters of Dlx1-expressing cells were detected in large numbers in all ventrolateral explants, which include the lateral ganglionic eminence, derived from either wild-type (15/15, Fig. 5D) or Ngn2 mutant (8/8, Fig. 5H) embryos. Dlx1 was also detected at high levels in medial (6/6, Fig. 5J) and dorsal (7/8, Fig. 5I) explants derived from Ngn2 mutant embryos but not in most medial $(13 / 15$, Fig. $5 F)$ and dorsal (12/13, Fig. 5E) explants dissected from wild-type embryos after 4 days in culture. Control medial and dorsal explants dissected from the contralateral telencephalic vesicle from wild-type embryos $(n=15$; Fig. $5 \mathrm{C})$ and Ngn2 mutants ( $n=8$; Fig. $5 \mathrm{G})$, fixed immediately after harvesting, did not express $D l \times 1$, confirming that ectopic Dlx1-expressing cells must have differentiated during the culture period. Thus, these data support the hypothesis that $D l \times 1^{+}$neurons in the Ngn2 mutant cortex are of dorsal origin and are mis-specified.

\section{Ngn2 functions as a neuronal determination gene in cortical lineages}

To determine to what extent the up-regulation of Mash1 compensates for the loss of Ngn2 function, we examined

Figure 5. Dlx1+ cortical neurons in Ngn2 mutants are of dorsal origin. $(A, B)$ Double labeling of frontal sections through the telencephalon of E12.5 Ngn2 $2^{\text {lacZKI }}$ homozygous mutant embryos with X-Gal (blue) and Dlx1 (purple) showing that ectopic $D l x 1$ neurons are $\mathrm{X}-\mathrm{Gal}^{+}$and thus originate from Ngn2-expressing dorsal telencephalic progenitors. $(C-K)$ Explants from E11 wild-type (top) and Ngn2 mutant (bottom) telencephalic vesicles dissected into ventral-lateral $(E, I)$, dorsal $(F, J)$, and medial $(G, K)$ domains, as defined in brackets
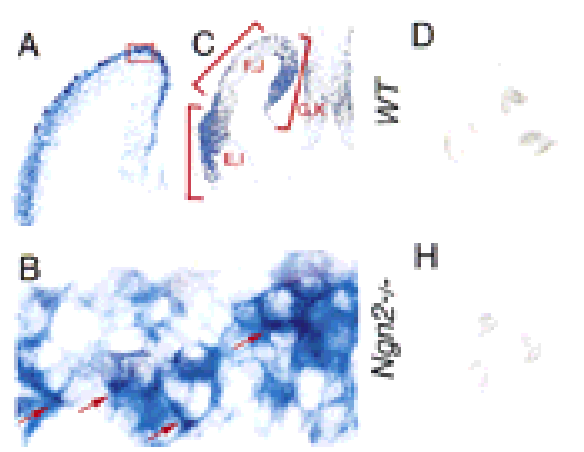
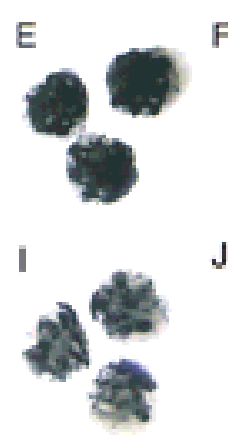

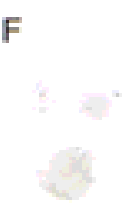

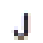

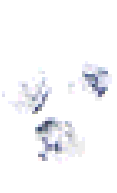

G

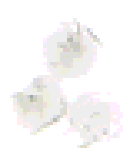

$\mathrm{K}$

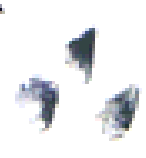

in $C$, cultured for 4 days on floating filters and labeled for Dlx1 transcripts. Explants of ventral-lateral domains derived from either wild-type $(E)$ or Ngn2 mutant $(I)$ embryos contained a large number of Dlx1+neurons. Dorsal $(F)$ and medial $(G)$ wild-type explants did not contain a significant number of $D l \times 1^{+}$neurons; in contrast, explants from the same domains of $N g n 2$ mutant embryos $(J$ and $K$, respectively) contained a large number of $D l \times 1^{+}$neurons (explants were defined as positive when more than three clusters were detected, each containing more than three $D l \times 1^{+}$cells). These neurons differentiate in dorsal explants isolated from ventral telencephalon and thus originate from dorsal progenitors. A small proportion of medial $(2 / 15)$ and dorsal (1/13) wild-type explants expressed Dlx1, possibly as a consequence of dissection errors. Confirmation that explants were dissected prior to the onset of ectopic Dlx1 expression was obtained from the lack of $D l x 1$ expression in uncultured contralateral dorsomedial explants from wild-type $(D)$ and Ngn2 mutant $(H)$ embryos. 
neurogenesis in the dorsal telencephalon of Ngn2;Mash1 double-mutant embryos.

The number of neurons expressing the panneuronal marker SCG10 is not significantly affected (Fig. 6B), and the neuronal differentiation gene NeuroD is normally expressed (Fig. 6E), in the cortex of Ngn2 single mutants at E12.5. As reported previously, no defect in neurogenesis is apparent in the dorsal telencephalon of Mash1 mutants (Casarosa et al. 1999; data not shown). In contrast, expression levels of SCG10 and NeuroD are strongly reduced in Ngn2;Mash1 mutant embryos (Fig. $6 \mathrm{C}, \mathrm{F})$, indicating that there is a reduction in number of PP neurons in double mutant embryos. Hes5, an E(spl)related gene activated by Delta/Notch signaling in the mouse CNS (de la Pompa et al. 1997; Ohtsuka et al. 1999 ) is expressed in the VZ throughout the telencephalon, and this expression is not affected in Ngn2 and Mash1 single mutants (Fig. 6G,H; data not shown). However, Hes5 expression is lost in the $\mathrm{VZ}$ of the dorsal/ medial cortex in Ngn2; Mash1 double mutants (Fig. 6I). Mash1 and Ngn2 are thus redundant for the activation of a downstream neuronal differentiation gene (NeuroD), the activation of Notch signaling (Hes5) and the generation of PP neurons in the dorsal/medial cortex (SCG10). These results indicate that Ngn2 normally has a determination function for early born cortical neurons, and that the up-regulation of Mash1 can partially compensate for the loss of this activity in Ngn2 mutants.

Mash1 up-regulation is required for the ventralization of Ngn2 mutant cortical neurons

Although reduced in number, neurons are present in the PP of Ngn2;Mash1 double mutant embryos, allowing us to assess whether the mis-specification of PP neurons in Ngn2 mutants is due to the loss of Ngn2 function or to the up-regulation of Mash1. We thus determined whether Mash1 is required for the ventralization of cortical neurons by examining Dlx1 expression in double mutant embryos. At E12.5, ectopic $D 1 \times 1+$ neurons were detected in the PP of Ngn2 single mutants (Fig. 6K), but not in Ngn2;Mash1 double mutant embryos (Fig. 6L), demonstrating that Mash1 activity is required for ectopic expression of Dlx1 in Ngn2 mutants. The reduction of expression of the dorsal-specific marker Math2 in Ngn2;Mash1 double mutants (Fig. 60) compared with wild types (Fig. 6M) parallels that of the general neuronal marker SCG10 (Fig. 6, cf. A and C), and double labeling with a Math2 RNA probe and an antibody to the panneuronal marker $\beta$-tubulin showed that $\beta$-tubulin+ neurons that remain in the dorsal telencephalon of Ngn2;Mash1 double mutant embryos maintain expression of Math2 and are thus correctly specified (data not shown). In contrast, the increase in the number of SCG10+ neurons in single Ngn2 mutants (Fig. 6B) compared with double Ngn2;Mash1 mutants (Fig. 6C) does not lead to a significant change in Math2 expression (Fig. 6 , cf. $\mathrm{N}$ and $\mathrm{O}$ ), suggesting that the additional cortical neurons resulting from Mash1 up-regulation in Ngn2

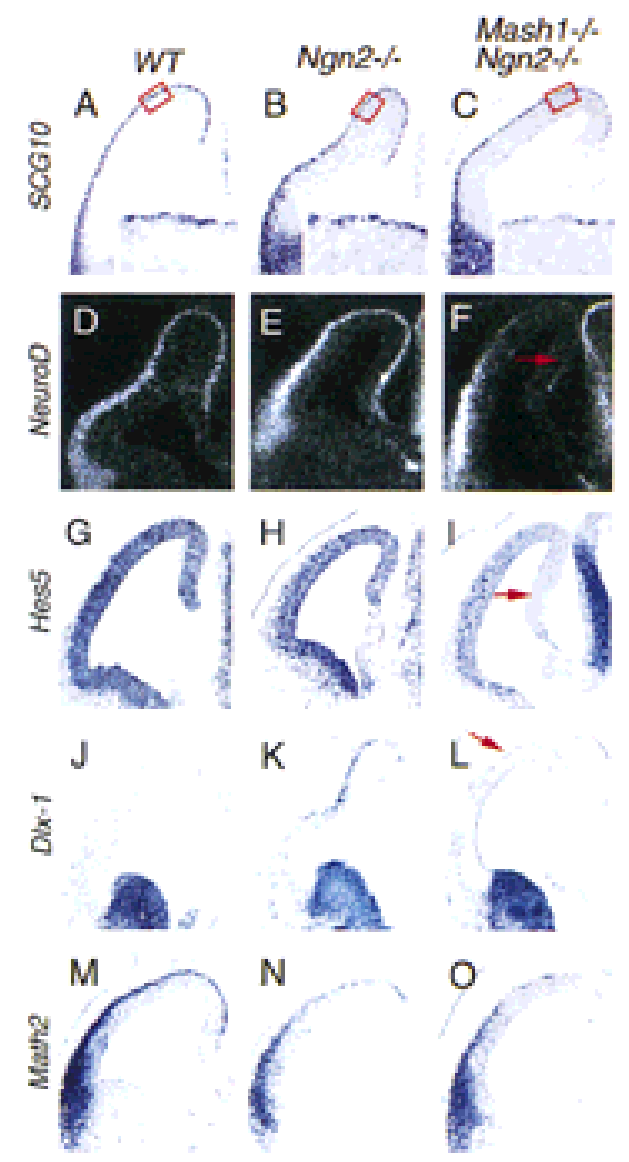

Figure 6. Up-regulation of Mash1 is required for the mis-specification of cortical neurons in Ngn2 mutants. $(A-C)$ Distribution of SCG10 transcripts in E12.5 wild-type, Ngn2 mutant, and Ngn2;Mash1 double mutant telencephalon. Neuronal loss is limited in the dorsal and medial PP of Ngn2 single mutants $(B)$ and more severe in Ngn2; Mash1 double mutants $(C)$. (D-F) Distribution of NeuroD transcripts in E12.5 wild-type, Ngn2 mutant, and Ngn2;Mash1 double mutant telencephalon. NeuroD expression is dramatically reduced in the dorsal and medial cortex of Ngn2;Mash1 double mutants (arrow, F) but is not affected in Ngn2 single mutants $(E)$. $(G-I)$ Distribution of Hes5 transcripts in E12.5 wild-type, Ngn2 mutant and Ngn2;Mash1 double mutant telencephalon. Hes5 expression is strongly down-regulated in the VZ of the dorsal and medial cortex of Ngn2;Mash1 double mutants (arrow, I), suggesting defects in Notch signaling in these progenitors. $(J-L)$ Distribution of Dlx1 transcripts in E12.5 wild-type, Ngn2 mutant, and Ngn2;Mash1 double mutant telencephalon. Ectopic $D l \times 1^{+}$neurons found in Ngn2 mutant cortex $(K)$ are absent in Ngn2;Mash1 double mutant embryos (arrow in L). Up-regulation of Mash1 is thus involved in the generation of $\mathrm{Dlx} 1^{+}$cortical neurons in Ngn2 mutants. $(M-O)$ Distribution of Math2 transcripts in E12.5 wild-type, Ngn2 mutant and, Ngn2;Mash1 double mutant telencephalon. The reduction in Math2 expression levels observed in the Ngn2 mutant cortex $(N)$ is also found in Ngn2;Mash1 double mutants $(O)$, thus confirming the overall reduction in number of medial and dorsal PP neurons in Ngn2;Mash1 double mutants.

mutants do not acquire a dorsal identity. Taken together, these results indicate that the up-regulation of Mash1 is 
required for the mis-specification of cortical neurons in Ngn2 mutants, including the ectopic expression of ventral markers and the down-regulation of dorsal markers.

\section{Forced Mash1 expression in dorsal progenitors is sufficient to induce ventral markers}

We then asked whether the up-regulation of Mash1 in dorsal progenitors is sufficient to ventralize cortical neurons, even in the context of Ngn2 expression. To address this question, we used homologous recombination in ES cells to generate a knock-in allele $\left(N g n 2^{\text {Mash1KI }}\right)$ in which Ngn2 coding sequences have been replaced by Mash1 (Fig. 7A). Heterozygous Ngn2 Mash1KI ES cells were used to generate chimeric embryos by aggregation with morula from the ROSA26 gene trap line in which lac $Z$ is ubiquitously expressed (Friedrich and Soriano 1991), allowing the distinction between wild-type and Ngn2 ${ }^{\text {Mash1KI }}$ cells. By use of this approach, dorsal telencephalic progenitors heterozygous for the Ngn2 Mash1KI allele should coexpress Ngn2 from the wild-type allele and Mash1 from the Ngn2 $2^{\text {Mash1KI }}$ allele. Chimeric embryos were sectioned and stained with X-Gal, and embryos in which mutant cells contributed nearly $100 \%$ to the dorsal telencephalon were analyzed further. As expected, a high level of Mash1 expression was observed in dorsal telencephalic progenitors of strongly chimeric embryos at E12.5 (data not shown) and E13.5 (Fig. 7E), as compared with wild-type embryos. As a result of the forced expression of Mash1 in Ngn2-expressing progenitors, ectopic expression of both Dlx1 (Fig. 7F) and GAD67 (Fig. 7G) was observed in cortical neurons, indicating that Mash1 is sufficient to induce ectopic expression of ventral markers in neurons of the dorsal telencephalon, even in the context of Ngn2 expression. This observation also supports the interpretation of the ex- periments in Figure 5, namely, that the presence of ventral-type neurons in the Ngn mutant cortex is the result of Mash1 up-regulation in dorsal progenitors, rather than of the migration of ventral neurons. However, embryos derived from ES cells heterozygous for the Ngn2 $2^{\text {Mash1KI }}$ allele differ from Ngn2 mutants in the reduced number and lateral, rather than dorsal, location of ectopic $D l \times 1^{+}$, $G A D 67^{+}$cells. Therefore, the loss of Ngn2 function appears to be also required for a more extensive ventralization of dorsal neurons.

\section{Discussion}

In this study, we have examined the role of the neural determination genes Ngn1, Ngn2, and Mash1 in specification of neuronal phenotypes in the telencephalon. We show that the normal restriction of Mash1 expression to ventral progenitors in the prospective basal ganglia is disrupted in Ngn2 single and Ngn1;Ngn2 double mutant embryos, indicating that one of the functions of the Ngns is to prevent Mash1 expression in dorsal progenitors. Using both Ngn2;Mash1 double mutant embryos and a new allele of Ngn2 in which Mash1 has been inserted into the locus, we demonstrate that although Mash1 can compensate to some extent for the neuronal determination function of Ngn2, it respecifies a subpopulation of neurons such that they express certain ventral-specific markers and lose expression of a number of dorsal-specific genes. Thus, genetic analyses have allowed us to uncover a novel role for Mash1 in the specification of ventral neuronal identities in the forebrain, as well as a role for the Ngns in the determination of cortical lineages. Taken together, these results provide new insights into how neurons with distinct phenotypes are born in different domains of the forebrain.
Figure 7. Forced expression of Mash1 in dorsal progenitors is sufficient to ventralize cortical neurons. (A) A new allele of Ngn2 was generated by replacing Ngn2 coding sequences by an IRES-Mash1 cassette $\left(N g n 2^{\text {Mash1KI }}\right)$. In this allele, Mash1 expression is controlled by Ngn2 regulatory sequences. Schematic representation of the endogenous Ngn2 locus (top), Ngn2 ${ }^{\text {Mash1KI }}$ targeting vector (middle), and recombined allele (bottom). Strongly chimeric E13.5 embryos generated with Ngn2 $2^{\text {Mash1KI }}$ heterozygous ES cells and a morula from the ROSA26 lacZ strain were analyzed. $(B, E)$ Distribution of Mash1 transcripts in the telencephalon of E13.5 wild-type and Ngn2 ${ }^{\text {Mash1KI }}$ chimeric embryos, demonstrating the up-regulation of Mash1 expression in dorsal progenitors of chimeric embryos (arrow, E). $(C, D, F, G)$ Distribution of Dlx1 and GAD67 transcripts in the telencephalon of E13.5 wild-type and Ngn2 ${ }^{\text {Mash1KI }}$ chimeric embryos, showing the presence of ectopic $D l \times 1+$ cells (arrow, F) and ectopic GAD67+ cells (arrow, G) in the lateral and dorsal PP of chimeric embryos.
A
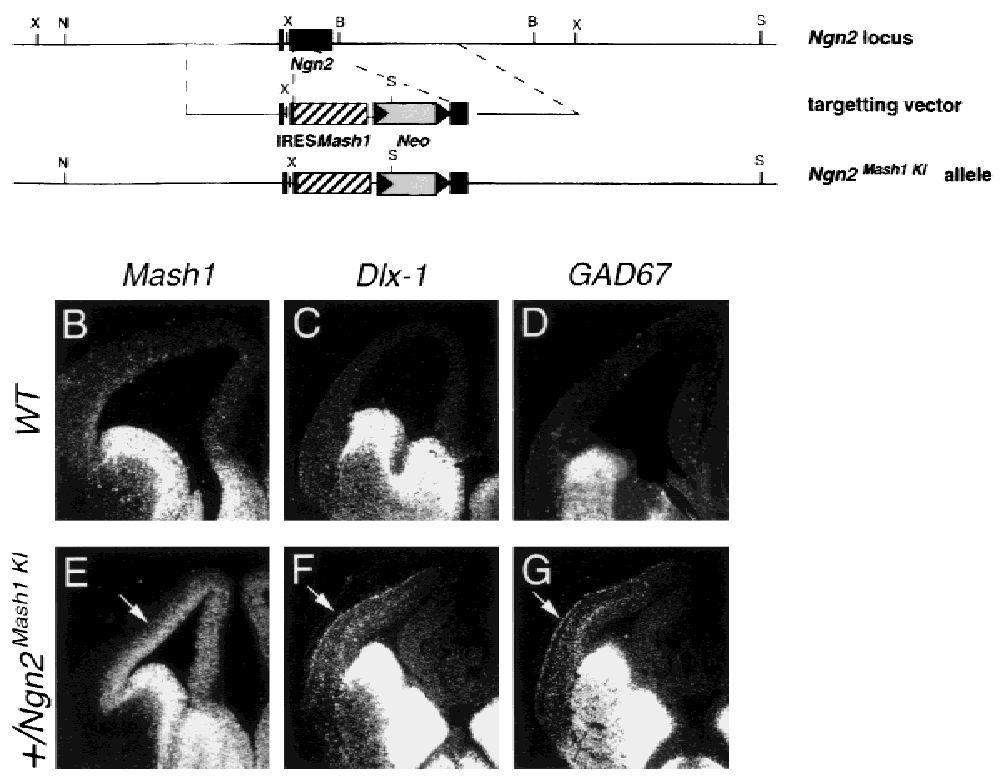
Ngns restrict Mash1 expression to ventral domains in the forebrain

Cross-regulatory interactions between neural determination genes have been reported previously in the PNS, in which Ngn1 and Ngn2 activate one another in complementary sets of cranial neuron precursors (Fode et al. 1998; Ma et al. 1998) and Ngn2 activates Ngn1 in a subset of precursors in the dorsal root ganglia ( $\mathrm{Ma}$ et al. 1999). In a similar manner, we show that Ngn1 expression in the dorsal/medial cortex is dependent on Ngn2 function. In contrast, $N g n 1$ is activated independently in the lateral cortex, and Ngn2 expression is, for the most part, independent of Ngn 1 throughout the cortex. Taken together with the higher levels of $N g n 2^{1 a c Z K I}$-driven $1 a c Z$ activity in the dorsal/medial VZ and higher levels of Ngn1 transcripts in the lateral VZ, these results indicate that Ngn genes are regulated in the cortex in a regionally restricted manner, suggesting an underlying heterogeneity of cortical progenitors. The unique identity of lateral cortical progenitors has been inferred previously from their early specification to produce neurons expressing latexin, a marker restricted to neurons of the lateral cortex (Arimatsu et al. 1999). Progenitors from other cortical regions have been shown to be similarly specified for expression of cortical area-specific markers (CohenTanouji et al. 1994; Levitt et al. 1997; Tole et al. 1997). However, only a few genes are expressed in a restricted manner in cortical progenitors that would reflect such a regional specification (i.e., Emx2; Gusilano et al. 1996). In this respect, the differential regulation of $N g n$ gene expression provides important molecular evidence for the early regional diversification of cortical progenitors.

Mash1 and the Ngns are expressed for the most part in a complementary manner in the forebrain (Gradwohl et al. 1996; Ma et al. 1997), respecting well-defined boundaries described previously for other regulatory molecules (Puelles and Rubenstein 1993; Rubenstein et al. 1998). We have demonstrated that one mechanism by which these complementary patterns are established and/or maintained is through the down-regulation of Mash1 expression in the dorsal telencephalon and dorsal thalamus by Ngn genes. Comparisons with other systems suggest that the establishment of the domains of neural determination gene expression is likely to be under the control of factors that pattern the early neural plate. For example, in the fly, expression of as-c genes in the wing imaginal disc is regulated by the homeobox proteins of the Iroquois complex and the GATA factor pannier, and ato is regulated in the eye by Shh signaling (GómezSkarmeta et al. 1996; Simpson 1996; Domínguez 1999), and similar interactions may be responsible for the initiation of Mash1 and Ngns expression in the mammalian forebrain. The regulation of Mash1 by the Ngns may represent a secondary mechanism involved in the refinement or the maintenance of these primary expression domains. In any case, our results show that cortical progenitors receive, and are competent to respond to, both Mash1- and Ngn-inducing signals. The repression of Mash1 by Ngns may be essential for the correct specifi- cation of cortical progenitors, by preventing Mash1 expression and thus ensuring that expression of Mash1 and $N g n$ genes remains mutually exclusive.

The mechanism by which Ngns suppress Mash1 expression in certain forebrain domains remains to be elucidated. One possibility is that a Notch-mediated process of lateral inhibition (Lewis 1996) or other non-cell autonomous mechanisms (Shou et al. 1999) are involved. Alternatively, the Ngns may function in a cell-autonomous manner to directly or indirectly repress transcription of Mash1. Additional experiments will be required to distinguish beween these hypotheses.

\section{Ngn2 functions as a neuronal determination gene in cortical lineages}

Previous studies have established that Ngn1 (Ma et al. 1998) and Ngn2 (Fode et al. 1998) function as neural determinants in the PNS, where they are required to activate a downstream bHLH regulatory cascade, to activate expression of Notch ligands and thereby initiate lateral inhibition, and to induce overt neuronal differentiation in subsets of sensory cranial ganglia. The idea that Ngn2 functions in a similar manner in cortical development comes primarily from the analysis of Mash1;Ngn2 double mutant embryos, in which the dorsal/medial cortex shows evidence of neural determination defects. In particular, this cortical region of double mutant embryos lacks expression of NeuroD, which is normally activated in immature CNS neurons as they leave the VZ (Lee et al. 1995), and which is induced in PNS neurons as part of a neuronal differentiation program activated by the $N g n$ genes (Fode et al. 1998; Ma et al. 1998) and by Mash1 (Cau et al. 1997). Expression of the Enhancer of splitrelated gene Hes5 is also down-regulated, suggesting that Delta/Notch signaling, which is required to activate expression of Hes5 in CNS progenitors (de la Pompa et al. 1997; Ohtsuka et al. 1999), is defective in Mash1;Ngn2 mutants. Finally, the number of neurons in the preplate of Mash1;Ngn2 mutants is drastically reduced. The neurogenesis defects in the Mash1;Ngn2 mutant cortex are not apparent in Ngn2 single or Ngn1;Ngn2 double mutants, demonstrating that Mash1, which is up-regulated in the dorsal VZ of these embryos, can compensate for the lack of Ngn gene function, activating Notch signaling and NeuroD expression and inducing cortical neuron differentiation. It is interesting to note that, in contrast to the dorsal telencephalon, the dorsal thalamus presents a severe deficit in neurons in Ngn1;Ngn2 double mutant embryos. This demonstrates that Ngn1 and Ngn2 have partially redundant determination functions in this tissue, and that Mash1 cannot compensate for the loss of $N g n s$, in spite of its up-regulation in the dorsal thalamus of Ngn1;Ngn2 mutants, and its neural determination function in the ventral thalamus (Torii et al. 1999). The basis for this difference between dorsal thalamus and dorsal telencephalon is presently unknown.

The neurogenesis defects seen in Mash1;Ngn2 mutants are restricted to the dorsal/medial cortex, most likely because the functions of Mash1, Ngn2, and Ngn1, 
which is regulated by Ngn2 in this region, are missing. In contrast, $N g n 1$ is expressed independently of $N g n 2$ in the lateral cortex, and is therefore likely to compensate for the lack of Ngn2 in this domain of the Mash1;Ngn2 mutant cortex. It is presently unknown whether Ngn1 and Ngn2 are involved in the development of distinct neuronal lineages in the lateral cortex, as proposed for sensory lineages in dorsal root ganglia (Ma et al. 1999), or whether the two genes function, and compensate for one another, within a unique population of progenitors in the lateral cortex.

\section{Mash1 ventralizes telencephalic neurons}

We have shown that up-regulation of Mash1 in the telencephalon of Ngn2, Ngn1;Ngn2, and Ngn2 Mash1KI mutants results in ectopic dorsal expression of $D l \times 1, D l \times 2$, Dlx5, and GAD67, indicating that Mash1 is able to specify dorsal progenitors to a more ventral fate. However, other ventral markers, including $L h \times 6, L h \times 7$, Isl1, and Nkx2.1, are not induced, which shows that Mash1 cannot specify all types of ventral telencephalic neurons, but only a subset of them. Neurons with a similar Dlx1/ $2^{+}, G A D 67^{+}$phenotype are normally found, albeit at a later stage (from E13.5 onward) and in smaller numbers, in the marginal and intermediate zones of the telencephalon. These neurons originate in their vast majority in the ganglionic eminences and reach the cortex by tangential migration (De Carlos et al. 1996; Anderson et al. 1997; Tamamaki et al. 1997; Lavdas et al. 1999; Zhu et al. 1999). In contrast, the ectopic Dlx1+ neurons in $\mathrm{Ngn}$ mutants originate from dorsal progenitors, which strongly suggests that Mash1 is able to respecify cortical progenitors to a new fate. However, it has been proposed that some GABAergic interneurons also differentiate from cortical progenitors, on the basis of results obtained in cortical explant cultures (Götz and Bolz 1994). Mash1, which is expressed at low levels in the VZ of the wildtype cortex, could thus be normally involved in the specification of these neurons, and up-regulation of Mash1 could result in a enlargement of this population of interneuron progenitors, leading to the precocious and excessive differentiation of GABAergic neurons of cortical origin. In both hypotheses, our results show that Mash1 is able to specify a particular neuronal identity, including the GABAergic neurotransmitter phenotype, in telencephalic neurons.

Does the phenotype of Mash1 null mutant mice support a role for Mash1 in neuronal type specification during normal telencephalic development? In these mice, subsets of ventral telencephalic neurons are missing, but $D 1 \times 1 / 2$ and GAD67 expression is maintained and there is no evidence for mis-specification among remaining ventral telencephalic neurons (Casarosa et al. 1999; Tuttle et al. 1999). One exception, however, is the population of migratory $D l \times 1 / 2+, G A D 67+$ cortical interneurons, which is greatly reduced in Mash1 mutants (Casarosa et al. 1999). Although it cannot be excluded that this defect is due to the loss of progenitors in the MGE and thus corresponds to the generic determination function of Mash1, it may also reflect a more specific function of Mash1 in the production of neurons with a $D l \times 1 / 2^{+}$, $G A D 67^{+}$neuronal phenotype. The reduction in GABAergic interneurons observed in Mash1 mutants appears to be more severe than that observed in Nkx2.1 mutants, in which there is a complete respecification of the MGE (Casarosa et al. 1999; Sussel et al. 1999; S. Casarosa and F. Guillemot, unpubl.), suggesting that Mash1 has additional functions in the production of these neurons beside the generation of MGE progenitors. Mash1 may thus be both sufficient and necessary to specify this particular ventral identity, whereas it is neither sufficient nor necessary for the specification of other ventral telencephalic phenotypes (i.e. expression of $N k x 2.1$, Is 11, Lhx6, Lhx7).

What could be the mechanisms by which Mash1 induces ventral-specific neuronal characteristics? Given that signaling by the secreted factor Sonic hedgehog (Shh) plays a major role in ventralization of the neural tube along its entire A/P axis (for review, see Ericson et al. 1997), including the forebrain (Barth and Wilson 1995; Ericson et al. 1995; Chiang et al. 1996; Hauptmann and Gerster 1996; Shimamura and Rubenstein 1997) it is tempting to hypothesize that Mash1 interacts with the Shh signaling pathway in the telencephalon. One possibility is that Mash1 specifies a ventral fate in telencephalic neurons by confering competence of telencephalic progenitors to respond to Shh. A precedent exists whereby Mash1 confers competence to neural crest progenitors to respond to the neuralizing activity of BMP2 (Lo et al. 1997). Competence to respond to Shh appears to be regulated and to change as telencephalic development proceeds, because early exposure of telencephalic explants to Shh induces expression of MGE-specific markers (Ericson et al. 1995), later exposure induces LGE-specific markers, whereas still later, telencephalic progenitors become refractory to induction by Shh (Kohtz et al. 1998). Up-regulation of Mash1 in dorsal progenitors could maintain the competence of these cells to respond to Shh and adopt a ventral fate, although they would have normally lost this competence at the time when Shh, which is expressed in ventral telencephalon throughout embryonic development (Miao et al. 1997), diffuses in the dorsal part of the telencephalon. However, only postmitotic neurons express ventral markers in the Ngn mutant cortex, and cortical progenitors remain correctly specified, which indicates that Mash1 would only control a late step in the specification of telencephalic progenitors by Shh.

Another possibility is that Mash1 is a downstream effector of Shh signaling in telencephalic progenitors. Expression of Mash1 could be induced by Shh in the ventral telencephalon as part of a program of ventral-type differentiation. Analogous situations are found in the Drosophila eye, in which the proneural gene atonal is activated by hedgehog (Domínguez 1999), and in somites in which the myogenic bHLH transcription factor MyoD is activated by Shh signaling (Münsterberg et al. 1995). Mash1 may thus function in a cell-autonomous manner to activate $D l \times 1 / 2$ expression and specify a GABAergic neurotransmitter phenotype in neuronal populations of 
the forebrain, possibly through direct activation of $D l x$ and GAD genes. The close correlation between expression of GAD67 and that of Dlx1/2 and Mash1 throughout the forebrain suggests a possible involvement of these transcription factors in specification of a GABAergic phenotype. In addition, the temporal sequence of their expression is consistent with the existence of a regulatory cascade between Shh, Mash1, and ventral-specific neuronal markers. For example, in the chick, Shh expression in the forebrain begins in the ventral diencephalon at stage 10 (Ericsson et al. 1995), whereas the chick homolog of Mash1, Cash1, is expressed in ventral telencephalon at stage 14 (Jasoni et al. 1995) and could therefore be induced by Shh acting from the ventral diencephalon in a planar manner. Expression of Cash1 precedes that of ventral neuronal markers such as Islet1, which is initiated in telencephalic neurons at about stage 17, suggesting that Cash1 could be involved in the activation of a ventral-specific program of differentiation in telencephalic neurons.

In hindbrain and autonomic neurons of the PNS, Mash1 is required for the differentiation of a noradrenergic neurotransmitter phenotype, possibly acting through regulation of the homeobox gene Phox2a (Hirsch et al. 1998; Lo et al. 1998). This suggests that, although Mash1 is likely to activate both generic and neuronal-type specific differentiation programs in various neuronal populations, it activates different programs in progenitors from different regions of the nervous system. Therefore, Mash1 activity is context dependent, as reported previously for Drosophila proneural genes (Jan and Jan 1994; Brunet and Ghysen 1999).

The study of Ngn2 and Ngn1;Ngn2 mutants did not allow us to firmly establish a role for the Ngns in specification of the dorsal identity of cortical neurons. Dorsalspecific markers are down-regulated in most, but not all, cortical neurons of $\mathrm{Ngn}$ mutants, suggesting that yet additional genes may partially compensate for the loss of Ngn genes and specify neurons with a dorsal character. As already seen for Mash1 in the ventral telencephalon (Casarosa et al. 1999), a loss-of-function analysis may not be sufficient to define the role of $\mathrm{Ngn}$ genes in specification of cortical neuron identities, and ectopic expression experiments, similar to those already performed in the PNS (Perez et al. 1999), may be necessary to examine this issue.

\section{Materials and methods}

Construction of $\mathrm{Ngn} 2^{\text {lacZKI }}$ and $\mathrm{Ngn} 2^{\mathrm{Mash} 1 / \mathrm{KI}}$ targeting vectors

The structure of the Ngn2 genomic locus was described previously (Fode et al. 1998). The Ngn2 $2^{\text {lacZKI }}$ and Ngn2 $2^{\text {Mash1/KI }}$ targeting vectors were both constructed with a 4-kb EagI fragment as the $5^{\prime}$ arm and a $1.5-\mathrm{kb}$ NheI fragment as the $3^{\prime}$ arm in a targeting vector containing a PGK-neo cassette flanked by loxP sites (Hanks et al. 1995). In both instances, the 5' and 3' UTR of $N g n 2$ was preserved, and an internal ribosomal entry site (IRES) element (Ghattas et al. 1991) was fused upstream of the lacZ or Mash1 coding sequences to ensure translational initiation.

\section{Generation of mutant and chimeric mice}

$N g n 2^{l a c Z K I}$ and $N g n 2^{\text {Mash1/KI }}$ targeting vectors were designed to replace the bHLH domain of Ngn2 with lacZ and Mash1 coding sequences, respectively, leaving 48 amino acids of amino-terminal Ngn2 coding sequence. Targeting vectors were electroporated into R1 ES cells by described conditions of culture, transfection, and selection of clones (Wurst and Joyner 1993). Genomic DNA was examined by Southern blotting to detect homologous recombination events with NotI-SpeI digests and $5^{\prime}$ and $3^{\prime}$ external probes that were described previously (Fode et al. 1998). For the Ngn2 ${ }^{\text {lacZKI }}$ allele, a recombinant ES cell clone was injected into C57BL/6J blastocysts and resulting chimeric males were bred with C57BL/6J females to obtain germ-line transmission of the mutation and the strain was maintained by crossing into a CD1 background. The loss of Ngn2 coding sequences, including the bHLH domain, in Ngn2 ${ }^{\text {lacZKI }}$ mutants was confirmed by PCR analysis with primers described in Fode et al. (1998) (data not shown). In addition, the similarity in phenotype of homozygous mutants bearing the original $\mathrm{Ngn} 2^{\text {neo }}$ null allele, which removed the entire Ngn2 coding sequence (Fode et al. 1998), and the Ngn2 ${ }^{\text {lacZKI }}$ allele confirmed that the new mutation is also a null allele (data not shown). For the Ngn2 $2^{\text {Mash1ki }}$ allele, chimeric embryos were generated by injecting 5-10 ES cells heterozygous for the mutation into morula stage (E2.5) embryos obtained from crossing males heterozygous for the ROSA26 gene trap insertion (Friedrich and Soriano 1991) with CD1 females and reimplanting embryos into pseudopregnant females. Chimeric embryos were harvested on day E12.5 or E13.5. Ngn1 (Ma et al. 1998), Ngn2 (Fode et al. 1998), and Mash1 (Guillemot et al. 1993) mutant mice have been described previously. To generate Ngn1;Ngn2 and Ngn2;Mash1 double homozygous mutant embryos, double heterozygous mice were intercrossed.

\section{Genotyping of Ngn1, Ngn2, and Ngn2 ${ }^{\text {lacZKI }}$ alleles}

Genotyping was performed by PCR on genomic DNA extracted from tails or embryonic yolk sacs as described previously (Fode et al. 1998). PCR genotyping of Mash1 (Guillemot et al. 1993), Ngn1 (Ma et al. 1998), and Ngn2 (Fode et al. 1998) mutant and wild-type alleles was as described previously. Genotyping of the Ngn2lacZKI allele was performed with primers situated in the upper (CCAGCTGGCGTAATAGCGAA) and lower (CGCCCGTTGCACCACAGATG) strands of the bacterial $\beta$-galactosidase sequence. PCR conditions for the Ngn2 $2^{\text {lacZKI }}$ allele were 30 cycles of $94^{\circ} \mathrm{C} / 1 \mathrm{~min} ; 60^{\circ} \mathrm{C} / 1 \mathrm{~min} ; 72^{\circ} \mathrm{C} / 1 \mathrm{~min}$.

\section{RNA in situ hybridization and lacZ staining}

The morning that vaginal plugs were observed is considered E0.5. Embryos were fixed at $4^{\circ} \mathrm{C}$ in $4 \%$ paraformaldehyde for 4 hr (E12.5) or overnight (E15.5 and older). Embryos were then rinsed in PBS, impregnated with $20 \%$ sucrose in PBS overnight at $4^{\circ} \mathrm{C}$, and embedded in OCT (Tissue-Tek, Miles). Sections were cut at $10 \mu \mathrm{m}$ on a cryostat. Nonradioactive and ${ }^{35}$ S-labeled section RNA in situ hybridization was performed as described in Cau et al. (1997). Double labeling nonradioactive in situs were performed with digoxygenin and fluorescein-labeled probes. Briefly, following the development of the NBT/BCIP reaction, the antidigoxygenin antibody was destroyed by a 15 min incubation in $0.1 \mathrm{M}$ glycine $(\mathrm{pH} 2.2)$. Sections were then washed three times for $5 \mathrm{~min}$ in PBS with $0.1 \%$ Tween 20 (PBT) and $10 \mathrm{~min}$ in MABT (100 mM maleic acid at $\mathrm{pH} 7.5,150 \mathrm{~mm}$ $\mathrm{NaCl}, 0.1 \%$ Tween 20). Sections were then blocked for $1 \mathrm{hr}$ in $2 \%$ Boehringer blocking reagent (BM 1096 176) made up in ma- 
leic acid buffer and $20 \%$ heat-inactivated goat serum followed by an overnight incubation in blocking solution with $1 / 1000$ dilution of anti-fluorescein antibody. Sections were then washed four to five times in MABT for $20 \mathrm{~min}$ and twice for 10 min in NMT (100 mM NaCl, $50 \mathrm{~mm} \mathrm{MgCl}_{2}, 100 \mathrm{~mm}$ Tris-Cl at $\mathrm{pH} 9.5$ ) and stained with $7.5 \mu \mathrm{l} / \mathrm{ml}$ INT in NMT buffer with 5 mm levamisole (D. Henrique, pers. comm.). $\beta$-Galactosidase staining was performed as described in Beddington et al. (1989) and double lacZ staining/RNA in situ hybridization was as described by Houzelstein and Tajbakhsh (1999). The probes used were described in the following publications: Ngn1 (Cau et al. 1997), Ngn2 (Gradwohl et al. 1996), Mash1 (Guillemot and Joyner 1993), Math2 (Bartholomä and Nave 1994; Shimizu et al. 1995), NeuroD (Lee et al. 1995); Hes5 (Akazawa et al. 1992), Dlx1 (Price et al. 1991), Dlx-5 (Simeone et al. 1994); SCG10 (Stein et al. 1988), GAD67 (Behar et al. 1994), and Reelin (Ikeda and Terashima 1997).

\section{Birthdating, immunohistochemistry, and histology}

For histological analyses, embryos were fixed in Bouin's fixative from $24 \mathrm{hr}$ (E12.5) up to 3 days (P0). From E15.5 to P0, brains were dissected from the skull prior to fixation. Brains were then processed for wax embedding and cut at $7 \mu \mathrm{m}$ and stained with hematoxylin-eosin or processed for anti-BrdU immunostaining. Biotinylated secondary anti-mouse and anti-rabbit immunoglobulin antibodies (1/200 dilution) and avidin-biotin complex reagents were from the Vectastain kit (Vector). For birthdating analysis, pregnant females were injected intraperitoneally with $2 \mathrm{mg}$ of BrdU (Sigma) either at E10.5, E11.5, E12.5, or E15.5 and sacrificed at E15.5 or P0. Embryos were either processed for wax sectioning for anti-BrdU immunohistochemistry or embedded in OCT for double RNA in situ/anti-BrdU immunostaining. The anti-BrdU (1/100 dilution; Boehringer Mannheim) staining was performed as described in Anderson et al. (1997).

\section{Telencephalic explants}

Telencephalic explants were dissected from E11.5 embryos and divided into dorsal, medial, and ventral-lateral domains essentially as described in Kohtz et al. (1998). Explants were cultured for 4 days on 0.02-mm Nucleopore filters floating on DMEM:F12 medium with $10 \%$ FCS. Explants were then fixed $1 \mathrm{hr}$ in $4 \%$ paraformaldehyde at $4^{\circ} \mathrm{C}$, rinsed two times in PBS and stored in methanol at $-20^{\circ} \mathrm{C}$. Wholemount RNA in situ hybridization was performed as described (Cau et al. 1997). High levels of Dlx1 expression were defined as greater than three groups of Dlx1expressing cells per explant.

\section{Acknowledgments}

We thank Jean-François Brunet, Christo Goridis, Magdalena Götz, Marion Wassef, and Masato Nakafuku for their critical comments on the manuscript. We also thank Magdalena Götz for helpful discussions, Aitana Perea and Muriel Rhinn for help with the generation of chimeras, and Domingos Henrique for advice on the double RNA in situ hybridization procedure. We also acknowledge the following people for their gifts of probes: Denis Duboule, Gérard Gradwohl, Peter Gruss, Marc Hallonet, Ryoichiro Kageyama, Dan Kaufman, and John Rubenstein. C.F. was supported by fellowships from the Human Frontiers Science Program and Medical Research Council of Canada and S.C. was supported by fellowships from the Association pour la Recherche sur le Cancer and Human Frontiers Science Program. This work was supported by grants from the European Com- mission Biotech Programme, Association pour la Recherche sur le Cancer, Association Française contre les Myopathies and Ministère de l'Enseignement et de la Recherche to F.G., and by institutional funds from INSERM, CNRS, and Hôpital Universitaire de Strasbourg.

The publication costs of this article were defrayed in part by payment of page charges. This article must therefore be hereby marked "advertisement" in accordance with 18 USC section 1734 solely to indicate this fact.

\section{References}

Akazawa, C., Y. Sasai, S. Nakanishi, and R. Kageyama. 1992. Molecular characterization of a rat negative regulator with a basic helix-loop-helix structure predominantly expressed in the developing nervous system. I. Biol. Chem. 26: 2187921885.

Anderson, D.J., and Y.N. Jan. 1997. The determination of the neuronal phenotype. In Molecular and cellular approaches to neural development (ed. W.M. Cowan), pp. 26-63. Oxford University Press, New York, NY.

Anderson, S.A., D.D. Eisenstat, L. Shi, and J.L.R. Rubenstein. 1997. Interneuron migration from basal forebrain to neocortex: Dependence on Dlx genes. Science 278: 474-476.

Arimatsu, Y., M. Ishida, K. Takiguchi-hayashi, and Y. Uratani. 1999. Cerebral cortical specification by early potential restriction of progenitor cells and later phenotype control of postmitotic neurons. Development 126: 629-638.

Barth, K.A. and S.W. Wilson. 1995. Expression of zebrafish $n k 2.2$ is influenced by sonic hedgehog/vertebrate hedgehog-1 and demarcates a zone of neuronal differentiation in the embryonic forebrain. Development 121: 1755-1768.

Bartholomä, A. and K.-A. Nave. 1994. NEX-1: A novel brain specific helix-loop-helix protein with autoregulation and sustained expression in mature cortical neurons. Mech. Dev. 48: $217-228$.

Beddington, R.S.P., J. Morgenstein, H. Land, and A. Hogan. 1989. An in situ transgenic enzyme marker for the midgestation mouse embryo and the visualization of the inner cell mass clones during early organogenesis. Development 106: 37-46.

Behar, T., W. Ma, L. Hudson, and J.L. Barker. 1994. Analysis of the anatomical distribution of GAD67 mRNA encoding truncated glutamic acid decarboxylase proteins in the embryonic rat brain. Dev. Brain Res. 77: 77-87.

Brunet, J.-F. and A. Ghysen. 1999. Deconstructing cell determination: Proneural genes and neuronal identity. BioEssays 21: 313-318.

Bulfone, A., S.M. Smiga, K. Shimamura, A. Peterson, L. Puelles, and J.L.R. Rubenstein. 1995. T-Brain-1: A homolog of Brachyury whose expression defines molecularly distinct domains within the cerebral cortex. Neuron 15: 63-78.

Casarosa, S., C. Fode, and F. Guillemot. 1999. Mash1 regulates neurogenesis in the ventral telencephalon. Development 126: 525-534.

Cau, E., G. Gradwohl, C. Fode, and F. Guillemot. 1997. Mash1 activates a cascade of bHLH regulators in olfactory neuron progenitors. Development 124: 1611-1621.

Cepko, C.L. 1999. The roles of intrinsic and extrinsic cues and bHLH genes in the determination of retinal cell fates. Curr. Opin. Neurobiol. 9: 37-46.

Chiang, C., Y. Litingtung, E. Lee, K.E. Young, J.L. Corden, H. Westphal, and P.A. Beachy. 1996. Cyclopia and defective axial patterning in mice lacking Sonic hedgehog gene function. Nature 383: 407-413. 
Chien, C.-T., C.-D. Shiao, L.Y. Jan, and Y.N. Jan. 1996. Neuronal type information encoded in the basic-helix-loop-helix domain of proneural genes. Proc. Nat1. Acad. Sci. 93: 1323913244.

Cohen-Tannoudji, M., C. Babinet, and M. Wassef. 1994. Early determination of a mouse somatosensory cortex marker. $\mathrm{Na}$ ture 368: 460-463.

De Carlos, J.A., L. López-Mascaraque, and F. Valverde. 1996. Dynamics of cell migration from the lateral ganglionic eminence in the rat. J. Neurosci. 16: 6146-6156.

de la Pompa, J.L., A. Wakeham, K.M. Correia, E. Samper, S. Brown, R.J. Aguilera, T. Nakano, T. Honjo, T.W. Mak, J. Rossant et al. 1997. Conservation of the Notch signaling pathway in mammalian neurogenesis. Development 124: $1139-1148$.

Domínguez, M. 1999. Dual role for Hedgehog in the regulation of the proneural gene atonal during ommatidia development. Development 126: 2345-2353.

Edlund, T. and T.M. Jessell. 1999. Progression from extrinsic to intrinsic signaling in cell fate specification: A view from the nervous system. Cell 96: 211-224.

Ericson, J., J. Muhr, M. Placzek, T. Lints, T.M. Jessell, and T. Edlund. 1995. Sonic Hedgehog induces the differentiation of ventral forebrain neurons: A common signal for ventral patterning within the neural tube. Cell 81: 747-756.

Ericson, J., J. Briscoe, P. Rashbass, V. van Heyningen, and T.M. Jessell. 1997. Graded sonic hedgehog signaling and the specification of cell fate in the ventral neural tube. Cold Spring Harb. Symp. Quant. Biol. 62: 451-466.

Figdor, M.C. and C.D. Stern. 1993. Segmental organization of embryonic diencephalon. Nature 363: 630-634.

Fishell, G. 1997. Regionalization in the mammalian telencephalon. Curr. Opin. Neurobiol. 7: 62-69.

Fode, C., G. Gradwohl, X. Morin, A. Dierich, M. LeMeur, C. Goridis, and F. Guillemot. 1998. The bHLH protein NEUROGENIN2 is a determination factor for epibranchial placode-derived sensory neurons. Neuron 20: 483-494.

Friedrich, G. and P. Soriano. 1991. Promoter traps in embryonic stem cells: A genetic screen to identify and mutate developmental genes in mice. Genes \& Dev. 5: 1513-1523.

Ghattas, I.R., J.R. Sanes, and J.E. Majors. 1991. The encephalomyocarditis virus internal ribosome entry site allows efficient coexpression of two genes from a recombinant provirus in cultured cells and in embryos. Mol. Cell. Biol. 11: 5848 5859.

Gomez-Skarmeta, J.L., R.D. del Corral, E. de la Calle-Mustienes, D. Rerre-Marco, and J. Modolell. 1996. Araucan and caupolican, two members of the novel iroquois complex, encode homeoproteins that control proneural and vein-forming genes. Cell 85: 95-105.

Goridis, C. and J.-F. Brunet. 1999. Transcriptional control of neurotransmitter phenotype. Curr. Opin. Neurobiol. 9: 4753.

Götz, M. and J. Bolz. 1994. Differentiation of transmitter phenotypes in rat cerebral cortex. Eur. J. Neurosci. 6: 18-32.

Gradwohl, G., C. Fode, and F. Guillemot. 1996. Restricted expression of a novel murine atonal-related bHLH protein in undifferentiated neural precursors. Dev. Biol. 180: 227-241.

Guillemot, F. and A.L. Joyner. 1993. Dynamic expression of the murine Achaete-Scute homologue Mash-1 in the developing nervous system. Mech. Dev. 42: 171-185.

Guillemot, F., L.-C. Lo, J.E. Johnson, A. Auerbach, D.J. Anderson, and A.L. Joyner. 1993. Mammalian achaete-scute homolog- 1 is required for the early generation of olfactory and autonomic neurons. Cell 75: 463-476.

Gulisano, M., V. Broccoli, C. Pardini, and E. Boncinelli. 1996.
Emx1 and Emx2 show different patterns of expression during proliferation and differentiation of the developing cerebral cortex in the mouse. Eur. J. Neurosci. 8: 1037-1050.

Hanks, M., W. Wurst, L. Anson-Cartwright, A.B. Auerbach, and A.L. Joyner. 1995. Rescue of the En-1 mutant phenotype by replacement of En-1 with En-2. Science 269: 679-682.

Hauptmann, G. and T. Gerster. 1996. Complex expression of the $z p-50$ pou gene in the embryonic zebrafish brain is altered by overexpression of sonic hedgehog. Development 122: $1769-1780$.

Hirsch, M.-R., M.-C. Tiveron, F. Guillemot, J.-F. Brunet, and C. Goridis. 1998. Control of noradrenergic differentiation and Phox2a expression by MASH1 in the central and peripheral nervous system. Development 125: 599-608.

Houzelstein, D. and S. Tajbakhsh. 1998. Increased in situ hybridization sensitivity using non-radioactive probes after staining for $\beta$-galactosidase activity. Technical Tips Online (In press).

Ikeda, Y. and T. Terashima. 1997. Expression of reelin, the gene responsible for the Reeler mutation, in embryonic development and adulthood in the mouse. Dev. Dyn. 210: 157-172.

Jan, Y.N. and L.Y. Jan. 1994. Genetic control of cell fate specification in Drosophila peripheral nervous system. Annu. Rev. Genet. 28: 373-393.

Jarman, A.P. and I. Ahmed. 1998. The specificity of proneural genes in determining Drosophila sense organ identity. Mech. Dev. 76: 117-125.

Jasoni, C.L., M.D. Walker, M.D. Morris, and T.A. Reh. 1994. A chicken achaete-scute homolog $(C A S H-1)$ is expressed in a temporally and spatially discrete manner in the developing nervous system. Development 120: 769-783.

Kohtz, J.D., D.P. Baker, G. Corte, and G. Fishell. 1998. Regionalization within the mammalian telencephalon is mediated by changes in responsiveness to Sonic hedgehog. Development 125: 5079-5089.

Lavdas, A.A., M. Grigoriou, V. Pachnis, and J.G. Parnavelas. 1999. The medial ganglionic eminence gives rise to a population of early neurons in the developing cereral cortex. $J$. Neurosci. 99: 7881-7888.

Lee, J.E. 1997. Basic helix-loop-helix genes in neural development. Curr. Opin. Neurobiol. 7: 13-20.

Lee, J.E., S.M. Hollenberg, L. Snider, D.L. Turner, N. Lipnick, and H. Weintraub. 1995. Conversion of Xenopus ectoderm into neurons by neuroD, a basic helix-loop-helix protein. Science 268: 836-844.

Levitt, P., M.F. Barbe, and K.L. Eagleson. 1997. Patterning and specification of the cerebral cortex. Annu. Rev. Neurosci. 20: $1-24$.

Lewis, J. 1996. Neurogenic genes and vertebrate neurogenesis. Curr. Opin. Neurobiol. 6: 3-10.

Lo, L., L. Sommer, and D.J. Anderson. 1997. MASH1 maintains competence for BMP2-induced neuronal differentiation in post-migratory neural crest cells. Curr. Biol. 7: 440-450.

Lo, L., M.C. Tiveron, and D.J. Anderson. 1998. MASH1 activates expression of the paired homeodomain transcription factor Phox2a, and couples pan-neuronal and subtype-specific components of auntomic neuronal identity. Development 125: 609-620.

Ma, Q., L. Sommer, P. Cserjesi, and D.J. Anderson. 1997. Mash1 and neurogenin 1 expression patterns define complementary domains of neuroepithelium in the developing CNS and are correlated with regions expressing Notch ligands. I. Neurosci. 17: 3644-3652.

Ma, Q., Z.F. Chen, I.B. Barrantes, J.L. de la Pompa, and D.J. Anderson. 1998. Neurogenin1 is essential for the determination of neuronal precursors for proximal cranial sensory gan- 
glia. Neuron 20: 469-482.

Ma, Q., C. Fode, F. Guillemot, and D.J. Anderson. 1999. Neurogenin 1 and neurogenin 2 are essential for the development of distinct subsets of sensory neurons in the dorsal root ganglia. Genes \& Dev. 13: 1717-1728.

Miao, N., M. Wang, J.A. Ott, J.S. D'Alessandro, T.M. Woolf, D.A. Bumcrot, N.K. Mahanthappa, and K. Pang. 1997. Sonic Hedgehog promotes the survival of specific CNS neuron populations and protects these cells from toxic insult in vitro. J. Neurosci. 17: 5891-5899.

Morrow, E.M., T. Furukawa, J.E. Lee, and C.L. Cepko. 1999. NeuroD regulates multiple functions in the developing neural retina in rodent. Development 126: 23-36.

Münsterberg, A.E., J. Kitajewski, D.A. Bumcrot, A.P. McMahon, and A.B. Lassar. 1995. Combinatorial signaling by Sonic hedgehog and Wnt family members induces myogenic bHLH gene expression in the somite. Genes \& Dev. 9: 2911-2922.

Neyt, C., M. Welch, A. Langston, J. Kohtz, and G. Fishell. 1997. A short-range signal restricts cell movement between telencephalic proliferative zones. J. Neurosci. 17: 9194-9203.

Ohtsuka, T., M. Ishibashi, G. Gradwohl, S. Nakanishi, F. Guillemot, and R. Kageyama. 1999. Hes1 and Hes5 as Notch effectors in mammalian neuronal differentiation. EMBO $\mathrm{J}$. 18: 2196-2207.

Parras, C., L. García-Alonso, I. Rodríguez, and F. Jiménez. 1996. Control of neural precursor specification by proneural genes in the CNS of Drosophila. EMBO J. 15: 6394-6399.

Perez, S.E., S. Rebelo, and D.J. Anderson. 1999. Early specification of sensory neuron fate revealed by expression and function of neurogenins in the chick embryo. Development 126: $1715-1728$.

Price, M., M. Lemaistre, M. Pischetola, R. Di Lauro, and D. Duboule. 1991. A mouse gene related to Distal-less shows a restricted expression in the developing forebrain. Nature 351: 748-751.

Puelles, L. and J.L.R. Rubenstein. 1993. Expression patterns of homeobox and other putative regulatory genes in the embryonic mouse forebrain suggests a neuromeric organization. Trends Neurosci. 16: 472-479.

Rubenstein, J.L.R., K. Shimamura, S. Martinez, and L. Puelles. 1998. Regionalization of the prosencephalic neural plate. Annu. Rev. Neurosci. 21: 445-477.

Shimamura, K. and J.L.R. Rubenstein. 1997. Inductive interactions direct early regionalization of the mouse forebrain. Development 124: 2709-2718.

Shimizu, C., C. Akazawa, S. Nakanishi, and R. Kageyama. 1995. MATH-2, a mammalian helix-loop-helix factor structurally related to the product of Drosophila proneural gene atonal, is specifically expressed in the nervous sytem. Eur. J. Biochem. 229: 239-248.

Shou, J., P.C. Rim, and A.L. Calof. 1999. BMPs inhibit neurogenesis by a mechanism involving degradation of a transcription factor. Nature Neurosci. 2: 339-345.

Simeone, A., D. Acampora, M. Pannese, M. D’Esposito, A. Stornaiuolo, M. Gulisano, A. Mallamaci, K. Kastury, T. Druck, K. Huebner et al. 1994. Cloning and characterization of two members of the vertebrate Dlx gene family. Proc. Natl. Acad. Sci. 15: 2250-2254.

Simpson, P. 1996. A prepattern for sensory organs. Curr. Biol. 6: $948-950$

Skeath, J.B. and C.Q. Doe. 1996. The achaete-scute complex proneural genes contribute to neural precursor specification in the Drosophila CNS. Curr. Biol. 6: 1146-1152.

Sommer, L., Q. Ma, and D.J. Anderson. 1996. neurogenins, a novel family of atonal-related bHLH transcription factors, are putative mammalian neuronal determination genes that reveal progenitor cell heterogeneity in the developing CNS and PNS. Mol. Cell. Neurosci. 8: 221-241.

Stein, R., N. Mori, K. Matthews, L.C. Lo, and D.J. Anderson. 1988. The NGF-inducible SCG10 mRNA encodes a novel membrane-bound protein present in growth cones and abundant in developing neurons. Neuron 1: 463-476.

Sussel, L., O. Marin, S. Kimura, and J.L.R. Rubenstein. 1999. Loss of Nkx2.1 homeobox gene function results in a ventral to dorsal molecular respecification within the basal telencephalon: Evidence for a transformation of the pallidum into the striatum. Development 126: 3359-3370.

Tamamaki, N., K.E. Fujimori, and R. Takauji. 1997. Origin and route of tangentially migrating neurons in the developing neocortical intermediate zone. J. Neurosci. 17: 8313-8323.

Tole, S., C. Christian, and E.A. Grove. 1997. Early specification and autonomous development of cortical fields in the mouse hippocampus. Development 124: 4959-4970.

Tomita, K., S. Nakanishi, F. Guillemot, and R. Kageyama. 1996. Mash1 promotes neuronal differentiation in the retina. Genes to Cells 1: 765-774.

Tori, M., F. Matsuzaki, N. Osumi, K. Kaibuchi, S. Nakamura, S. Casarosa, F. Guillemot, and M. Nakafuku. 1999. Transcription factors Mash1 and Proxl delineate early steps in differentiation of neural stem cells in the developing central nervous system. Development 126: 443-456.

Tuttle, R., Y. Nakagawa, J.E. Johnson and D.D.M. O'Leary. 1999. Defects in thalamocortical axon pathfinding correlate with altered cell domains in Mash1-deficient mice. Deve1opment 124: 4959-4970.

Wurst W. and A.L. Joyner. 1993 Production of targeted embryonic stem cell clones. In Gene targeting, a practical approach, (ed. A.L. Joyner), pp. 33-61. Oxford University Press, Oxford, UK.

Zhu, Y., H.-S. Li, L. Zhou, J.Y. Wu, and Y. Rao. 1999. Cellular and molecular guidance of GABAergic neuronal migration from an extracortical origin to the neocortex. Neuron 23: 473-485. 


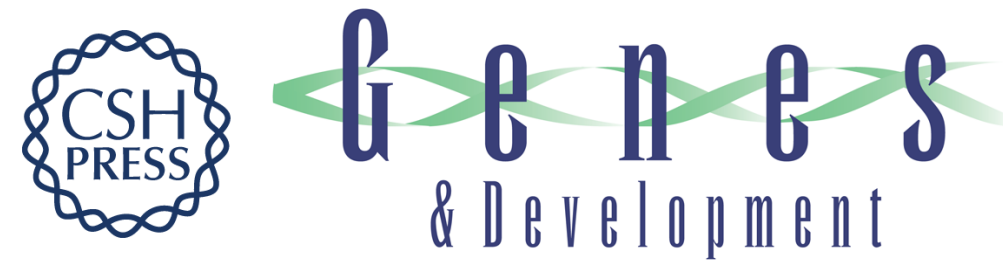

\section{A role for neural determination genes in specifying the dorsoventral identity of telencephalic neurons}

Carol Fode, Qiufu Ma, Simona Casarosa, et al.

Genes Dev. 2000, 14:

Access the most recent version at doi:10.1101/gad.14.1.67

$\begin{array}{ll}\text { References } & \begin{array}{l}\text { This article cites } 73 \text { articles, } 33 \text { of which can be accessed free at: } \\ \text { http://genesdev.cshlp.org/content/14/1/67.full.html\#ref-list-1 }\end{array}\end{array}$

License

Email Alerting Receive free email alerts when new articles cite this article - sign up in the box at the top Service right corner of the article or click here.

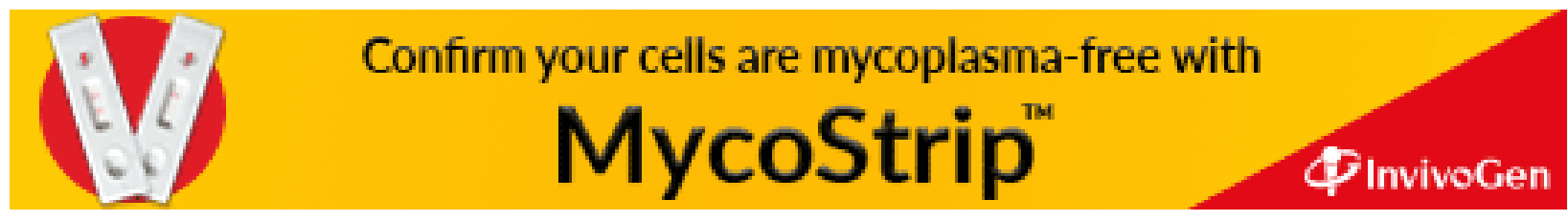

